\title{
GEAR-RACK TOOL FOR GENERATING AN INVOLUTE FLANK. VIRTUAL POLE METHOD
}

\author{
Georgiana Alexandra MOROȘANU 1, Virgil Gabriel TEODOR 1, \\ Nicolae OANCEA 1 \\ ${ }^{1}$ Department of Manufacturing Engineering, "Dunărea de Jos” University of Galați, România \\ email: Alexandra.Costin@ugal.ro
}

\begin{abstract}
In the paper, an approach to the issue of gear-rack type tool profiling is proposed, based on the "virtual pole" method. The proposed issue has also been solved based on complementary fundamental theorems, but the virtual pole method has the advantage of a simplified form of writing the generating movements. This makes it possible to avoid the need to write the relative movements between the tool and the piece. The paper presents the application of the virtual pole method for generating an involute profile.
\end{abstract}

KEYWORDS: gear-rack tool, involute flank, virtual pole, enwrapping condition.

\section{INTRODUCTION}

In most cases, rolling generating is based on the kinematics of the machining process, which is determined by the kinematic chain of the machine tool on which the processing is performed [1].

Tools profiling for the rolling generating of vortices of surfaces with radial symmetry can be done by applying various analytical [2] or graphical [3-7] methods.

The analytical methods for determining the enwrapping condition can be grouped into fundamental methods [2] or complementary methods [3].

Regardless of the type of method used, the analytical profiling of the generating tool involves following an algorithm composed of the following steps:

- Identifying the profile to be generated. This profile can be known in analytical form, through its parametric equations, or in discrete form, through coordinates of some points that belong to it.

- Writing the equations of absolute movements executed by tool and blank during generating.

- Writing the equations of relative movements that occur between the tool and the blank.

- Finding the enveloping condition and identifying the tool profile based on it.

The mentioned steps involve writing complicated equations, which can generate errors and often require a high volume of mathematical calculations. To avoid this, an alternative method for determining the profile of the generating tool was imagined, a method that was called the virtual pole method [4].

This method eliminates the need to write the equations of relative movement between the tool and the blank, this movement being replaced by the successive positioning of the points on the piece profile in the space of the fixed reference system, as points belonging to the contact curve and then in the space of the reference system associated with the tool, as points belonging to its profile.

The method is based on the fundamental theorem known as the normal theorem and which states that: ,in the case of two reciprocal enwrapping profiles, associated with a couple of rolling centrodes, they admit at the contact point a common normal which passes through the gearing pole". The gearing pole is defined as the tangency point of the two centrodes.

Based on this theorem, it can be observed that a virtual pole of gearing can be imagined, defined as intersection between the normal taken to the profile to be generated and the centrode associated with it and which, under certain conditions, can become the real gearing pole. Finding these conditions allows the identification of the enwrapping condition and can be done by changing the position of the blank until the virtual pole overlaps the contact point of the two centrodes.

In this position, the normal theorem is observed so the point considered on the piece profile is in contact with a point on the tool profile.

Various applications have been created for profiling tools such as rack, rotary cutter, gear-shaped cutter, for generating elementary profiles, such as the 
straight segment or circle spring, known in analytical form.

The paper presents the application of the virtual pole method for generating a profile frequently used in the technique: the involute profile.

\section{THE PROFILING ALGORITHM}

Figure 1 shows the gear-rack tool for generating an involute flank, where $\Delta$ represents the line that generates the involute curve; $\Sigma$ - the curve taken to the profile to be generated.

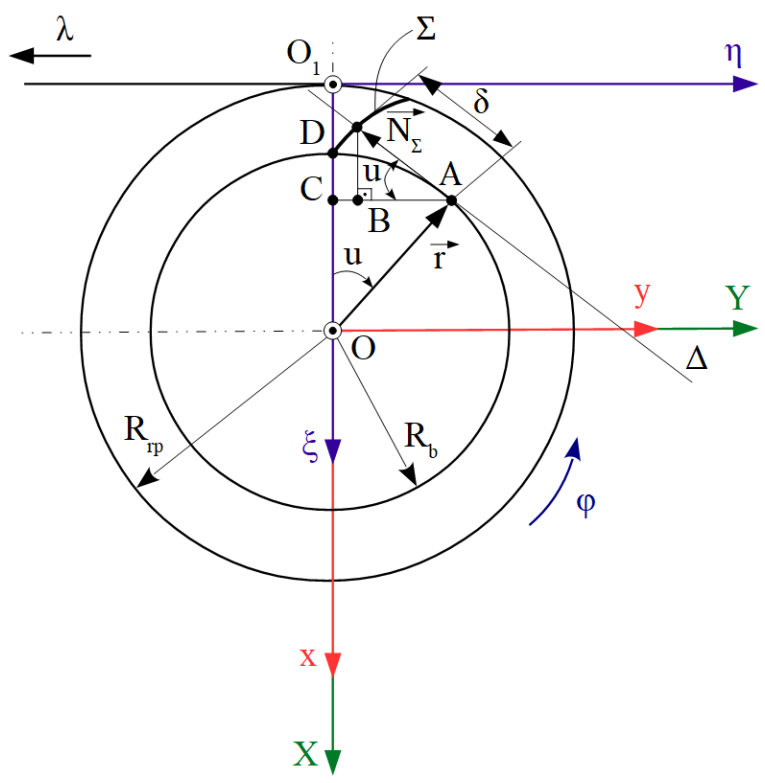

Fig. 1. Gear-rack tool for generating an involute flank

The equations of the involute profile will be:

$$
\Sigma: \mid \begin{aligned}
& X(u)=-R_{b} \cdot \cos u-R_{b} \cdot u \cdot \sin u ; \\
& Y(u)=R_{b} \cdot \sin u-R_{b} \cdot u \cdot \cos u .
\end{aligned}
$$

where $R_{b}$ is the radius of the base circle.

The absolute movement of the piece is given by:

$$
x=\omega_{3}^{T}(\varphi) \cdot X,
$$

or, developed:

$$
\begin{aligned}
& \left(\begin{array}{l}
x \\
y
\end{array}\right)=\left(\begin{array}{cc}
\cos \varphi & -\sin \varphi \\
\sin \varphi & \cos \varphi
\end{array}\right) \cdot\left(\begin{array}{c}
-R_{b} \cdot \cos u-R_{b} \cdot u \cdot \sin u \\
R_{b} \cdot \sin u-R_{b} \cdot u \cdot \cos u
\end{array}\right)= \\
& =\left(\begin{array}{l}
-R_{b} \cdot \cos u \cdot \cos \varphi-R_{b} \cdot u \cdot \sin u \cdot \cos \varphi- \\
-R_{b} \cdot \cos u \cdot \sin \varphi-R_{b} \cdot u \cdot \sin u \cdot \sin \varphi+ \\
-R_{b} \cdot \sin u \cdot \sin \varphi+R_{b} \cdot u \cdot \cos u \cdot \sin \varphi \\
+R_{b} \cdot \sin u \cdot \cos \varphi-R_{b} \cdot u \cdot \cos u \cdot \cos \varphi
\end{array}\right)= \\
& =\left(\begin{array}{l}
-R_{b} \cdot(\cos u \cdot \cos \varphi+\sin u \cdot \sin \varphi)- \\
R_{b} \cdot(\sin u \cdot \cos \varphi-\cos u \cdot \sin \varphi)- \\
-R_{b} \cdot u \cdot(\sin u \cdot \cos \varphi-\cos u \cdot \sin \varphi) \\
-R_{b} \cdot u \cdot(\cos u \cdot \cos \varphi+\sin u \cdot \sin \varphi)
\end{array}\right) \Rightarrow
\end{aligned}
$$

$$
\Rightarrow \mid \begin{aligned}
& x=-R_{b} \cdot \cos (u-\varphi)-R_{b} \cdot u \cdot \sin (u-\varphi) ; \\
& y=R_{b} \cdot \sin (u-\varphi)-R_{b} \cdot u \cdot \cos (u-\varphi) .
\end{aligned}
$$

The absolute movement of the tool is given by:

$$
x=\xi+A ; \quad A=\left(\begin{array}{c}
-R_{r p} \\
-R_{r p} \cdot \varphi
\end{array}\right),
$$

and from the rolling condition:

$$
\lambda=R_{r p} \cdot \varphi
$$

where: $R_{r p}$ represents the rolling radius of the piece and $\varphi-$ the rotation angle of the $X O Y$ system.

The normal to $\Sigma$ profile at the current point $M$ is given by:

$$
\overrightarrow{N_{\Sigma}}=\left(X(u)+\dot{Y}_{u} \cdot \delta\right) \cdot \vec{i}+\left(Y(u)-\dot{X}_{u} \cdot \delta\right) \cdot \vec{j},
$$

where $\delta$ represents a variable scalar in direction of normal.

Developing the equation (6) is obtained:

$$
\begin{aligned}
& \overrightarrow{N_{\Sigma}}=\left(-R_{b} \cdot \cos u-R_{b} \cdot u \cdot \sin u+\right. \\
& \left.+R_{b} \cdot u \cdot \delta \cdot \sin u\right) \cdot \vec{i}+ \\
& +\left(R_{b} \cdot \sin u-R_{b} \cdot u \cdot \cos u+\right. \\
& \left.+R_{b} \cdot u \cdot \delta \cdot \cos u\right) \cdot \vec{j} .
\end{aligned}
$$

Intersecting the normal $\overrightarrow{N_{\Sigma}}$ with the piece centrode, the circle of radius $R_{r p}$, having the equations:

$$
C_{1}: \mid \begin{aligned}
& X=-R_{r p} \cdot \cos \varphi \\
& Y=R_{r p} \cdot \sin \varphi
\end{aligned}
$$

is obtained:

$$
\mid \begin{aligned}
& -R_{b} \cdot \cos u-R_{b} \cdot u \cdot \sin u \cdot(1-\delta)=-R_{r p} \cdot \cos \varphi \\
& R_{b} \cdot \sin u-R_{b} \cdot u \cdot \cos u \cdot(1-\delta)=R_{r p} \cdot \sin \varphi
\end{aligned}
$$

In the previous equations system, the parameter $\delta$ is eliminated:

$$
\begin{aligned}
& 1-\delta=\frac{R_{r p} \cdot \cos \varphi-R_{b} \cdot \cos u}{R_{b} \cdot u \cdot \sin u}= \\
& =\frac{-R_{r p} \cdot \sin \varphi+R_{b} \cdot \sin u}{R_{b} \cdot u \cdot \cos u} \Rightarrow \\
& \Rightarrow R_{r p} \cdot \cos \varphi \cdot u \cdot \cos u-R_{b} \cdot u \cdot \cos ^{2} u+ \\
& +R_{r p} \cdot \sin \varphi \cdot u \cdot \sin u-R_{b} \cdot u \cdot \sin ^{2} u=0 ; \\
& R_{r p} \cdot u \cdot(\cos u \cdot \cos \varphi+\sin u \cdot \sin \varphi)= \\
& =R_{r p} \cdot u \cdot\left(\sin ^{2} u \cdot \cos ^{2} u\right) \Rightarrow \\
& \Rightarrow R_{r p} \cdot u \cdot \cos (u-\varphi)=R_{b} \cdot u .
\end{aligned}
$$


For $u \neq 0$ results the equation for determining the enwrapping condition:

$$
\cos (u-\varphi)=\frac{R_{b}}{R_{r p}} \Rightarrow \varphi=\arccos \left(\frac{R_{b}}{R_{r p}}\right)+u .
$$

The profile of the generating tool is obtained by associating to the tool movement equations with the enveloping condition.

Concretely, for a certain value of the parameter „u", the value of the angle , $\varphi$ ", with which the piece must be rotated, is calculated so that the virtual pole superimposes on the gearing pole:

$$
\varphi_{u}=\arccos \left(\frac{R_{b}}{R_{r p}}\right)+u .
$$

In this position, the current point is in tangent to the profile to be generated of the tool, so it is also on the contact line.

The coordinates of the current point, in the fixed reference system, will be, for $u=u_{M}$ :

$M: \mid \begin{aligned} & x_{M}=-R_{b} \cdot \cos \left(u_{M}-\varphi_{u}\right)-R_{b} \cdot u_{M} \cdot \sin \left(u_{M}-\varphi_{u}\right) ; \\ & y_{M}=R_{b} \cdot \sin \left(u_{M}-\varphi_{u}\right)-R_{b} \cdot u_{M} \cdot \cos \left(u_{M}-\varphi_{u}\right) .\end{aligned}$

Considering that at this moment the tool is moved according to the $\varphi_{u}$ value, the current point coordinates in the tool system will be:

$$
M \mid \begin{aligned}
& \xi_{M}=x_{M}+R_{r p} \\
& \eta_{M}=y_{M}+R_{r p} \cdot \varphi_{u}
\end{aligned}
$$

For $u=u_{\text {min }} \rightarrow u_{\text {max }}$, the $S$ profile of tool is obtained.

\section{APPLICATION}

The profile of the generating tool $S$ is calculated, having the input data presented in table 1.

Table 1. Input data for the numerical application

\begin{tabular}{|c|c|}
\hline $\mathbf{R}_{\mathbf{b}}[\mathbf{m m}]$ & 50 \\
\hline $\mathbf{R}_{\mathbf{r p}}[\mathbf{m m}]$ & 52 \\
\hline $\mathbf{u}_{\min }[\mathbf{r a d}]$ & 0 \\
\hline $\mathbf{u}_{\max }[\mathbf{r a d}]$ & 0.262 \\
\hline
\end{tabular}

The numerical results are presented in table 2 and the graphical representation of the gear-rack profile is shown in figure 2.
Table 2. Coordinates of points on the gear-rack tool profile

\begin{tabular}{|c|c|c|}
\hline Crt. no. & $\xi[\mathbf{m m}]$ & $\boldsymbol{\eta}[\mathbf{m m}]$ \\
\hline $\mathbf{1}$ & 3.929 & 0.709 \\
\hline $\mathbf{2}$ & 4.081 & 0.832 \\
\hline $\mathbf{3}$ & 4.249 & 0.884 \\
\hline $\mathbf{4}$ & 4.417 & 0.935 \\
\hline $\mathbf{5}$ & 4.585 & 0.986 \\
\hline $\mathbf{6}$ & 4.753 & 1.037 \\
\hline $\mathbf{7}$ & 4.921 & 1.089 \\
\hline $\mathbf{8}$ & 5.089 & 1.140 \\
\hline $\mathbf{9}$ & 5.257 & 1.191 \\
\hline $\mathbf{1 0}$ & 5.425 & 1.242 \\
\hline $\mathbf{1 1}$ & 5.593 & 1.293 \\
\hline $\mathbf{1 2}$ & 5.761 & 1.345 \\
\hline $\mathbf{1 3}$ & 5.929 & 1.396 \\
\hline $\mathbf{1 4}$ & 6.097 & 1.447 \\
\hline $\mathbf{1 5}$ & 6.265 & 1.498 \\
\hline $\mathbf{1 6}$ & 6.433 & 1.550 \\
\hline $\mathbf{1 7}$ & 6.601 & 1.601 \\
\hline $\mathbf{1 8}$ & 6.769 & 1.652 \\
\hline $\mathbf{1 9}$ & 6.937 & 1.703 \\
\hline $\mathbf{2 0}$ & 7.105 & 1.754 \\
\hline $\mathbf{2 1}$ & 7.273 & 1.806 \\
\hline & & \\
\hline
\end{tabular}

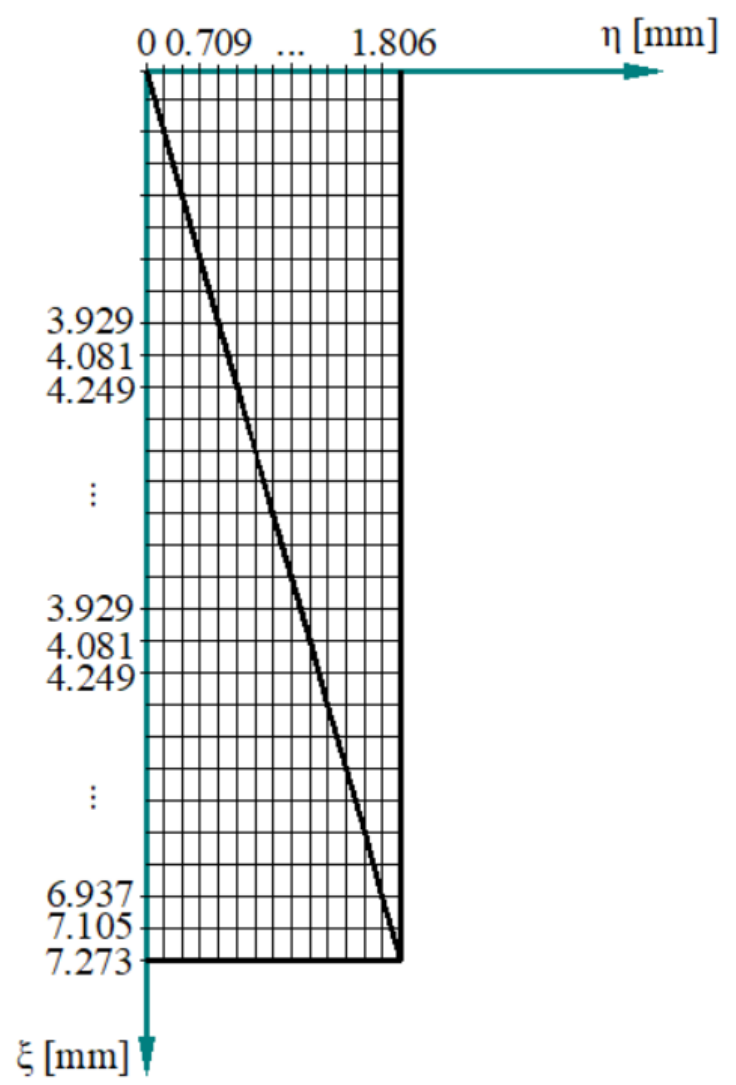

Fig. 2. Gear-rack tool profile 


\section{CONCLUSIONS}

The paper presents an alternative method for determining the profile of the generating tool, a method that has been called the ,virtual pole method".

The method eliminates the need to write the equations of relative movement between the tool and the blank, which substantially reduces the volume of calculations required and eliminates a significant source of possible errors.

The paper presents the application of the virtual pole method for generating an involute profile. This type of profile was chosen on the grounds that it is common in practice, being also a classic example of a profile obtained by enwrapping using the rolling method.

\section{ACKNOWLEDGEMENTS}

This work is supported by the project ANTREPRENORDOC, in the framework of Human Resources Development Operational Programme 2014-2020, financed from the European Social Fund under the contract number 36355/23.05.2019 HRD OP /380/6/13 - SMIS Code: 123847.

\section{REFERENCES}

[1] Radzevich, S., Kinematic Geometry of Surface Machining, CRC Press, Boca Raton, London, ISBN 978-1-4200-6340-0, 2007;

[2] Oancea, N., Surface Generation through Winding, Fundamental Theorems, vol. 1, "Dunarea de Jos" University Foundation Publishing House, ISBN 673-627-106-4, 2003.

[3] Oancea, N., Surface Generation through Winding, Complementary Theorems, vol. 2, "Dunarea de Jos" University Foundation Publishing House, ISBN 673-627-106-4, 2004;

[4] Costin, G.A., Teodor, V.G., Oancea, N., Virtual pole method - alternative method for profiling tools which generate by enwrapping, TMB, ISSN 2668-4829 (print), 2668-4888 (online), 31-34, 2019;

[5] Teodor, V.G., Costin, G.A., Virtual pole method alternative method for profiling rack tools, TMB, ISSN 2668-4829 (print), 2668-4888 (online), 5-8, 2019;

[6] Teodor, V.G., Baroiu, N., Susac, F., The Synthesis of New Algorithms for CAD Profiling of Cutting Tools, Lambert Academic Publishing, ISBN 978-613-7-08923-1, 2018. 